ff. M. Fisher • M. E. Trujillo • W. Hanif • A. H. Barnett •

P. G. McTernan · P. E. Scherer · S. Kumar

\title{
Serum high molecular weight complex of adiponectin correlates better with glucose tolerance than total serum adiponectin in Indo-Asian males
}

Received: 2 December 2004 / Accepted: 11 January 2005 / Published online: 19 May 2005

C) Springer-Verlag 2005

\begin{abstract}
Aims/hypothesis: It is well established that total systemic adiponectin is reduced in type 2 diabetic subjects. To date most studies have been concerned with the singular full-length protein or proteolytically cleaved globular domain. It is, however, apparent that the native protein circulates in serum as a lower molecular weight hexamer and as larger multimeric structures of high molecular weight (HMW). In this study we address the clinical significance of each form of the protein with respect to glucose tolerance. Methods: Serum was obtained from 34 Indo-Asian male subjects (BMI 26.5 \pm 3.1 ; age $52.15 \pm 10.14$ years) who had undertaken a 2-h oral glucose tolerance test. An aliquot of serum was fractionated using velocity sedimentation followed by reducing SDS-PAGE. Western blots were probed for adiponectin, and HMW adiponectin as a percentage of total adiponectin (percentage of higher molecular weight adiponectin $\left[S_{\mathrm{A}}\right]$ index) was calculated from densitometry readings. Total adiponectin was measured using ELISA; leptin, insulin and IL-6 were determined using ELISA. Results: Analysis of the cohort demonstrated that total adiponectin $(r=0.625, p=0.0001)$, fasting insulin $(r=-0.354$, $p=0.040)$ and age $(r=0.567, p=0.0001)$ correlated with $S_{\mathrm{A}}$. $S_{\mathrm{A}}$ showed a tighter, inverse correlation with 2-h glucose
\end{abstract}

ff. M. Fisher · P. G. McTernan · S. Kumar $(\bowtie)$

Unit for Diabetes and Metabolism, Warwick Medical School, Clinical Sciences Research Institute, University of Warwick,

UHCW Campus, Clifford Road,

CV2 2DX Coventry, UK

e-mail: Sudhesh.Kumar@warwick.ac.uk

Tel.: +44-247-6574665

Fax: $+44-247-6574871$

M. E. Trujillo · P. E. Scherer

Department of Cell Biology and Diabetes Research and

Training Centre, Albert Einstein College of Medicine,

Bronx, NY, USA

W. Hanif · A. H. Barnett

Department of Diabetes and Endocrinology,

Birmingham Heartlands Hospital,

Birmingham, UK levels $(r=-0.58, p=0.0003)$ than total adiponectin $(r=-0.38$, $p=0.0001)$. Conclusions/interpretation: This study demonstrates the importance of the $S_{\mathrm{A}}$ index as a better determinant of glucose intolerance than measurements of total adiponectin. Our findings suggest that HMW adiponectin is the active form of the protein.

Keywords Adipokines - Adiponectin - Glucose tolerance $\cdot$ Higher molecular weight species - Obesity · Type 2 diabetes mellitus

Abbreviations HMW: higher molecular weight - LMW: lower molecular weight $\cdot S_{\mathrm{A}}$ : percentage of higher molecular weight adiponectin

\section{Introduction}

Type 2 diabetes mellitus is associated with insulin resistance, impaired glucose tolerance and subclinical inflammation. Several factors are thought to contribute to the development of this disease, including age, ethnicity, family history and in particular increased adiposity. While the molecular mechanisms that link obesity with diabetes remain unclear, several studies to date have shown that adipocytes secrete factors, referred to as 'adipokines', which may contribute to the risk of diabetes.

Adiponectin (Acrp30, apm1, gbp28) a 30-kDa protein, is secreted from human mature adipocytes and has been shown to be an important regulator of glucose and lipid metabolism. Serum adiponectin levels are known to be reduced in both childhood and adult obesity $[1,2]$. Decreased serum adiponectin can serve as a potent predictor of the future development of insulin resistance, type 2 diabetes and cardiovascular disease [3, 4]. In addition, the human adiponectin gene has been mapped to locus $3 \mathrm{q} 27$, which has been identified as a susceptibility locus for type 2 diabetes and the metabolic syndrome [5]. Although the elucidation of the molecular mechanisms by which adiponectin exerts its insulin-sensitising effects is still the subject of intense research, several lines of evidence point to an essential role 
of AMP kinase as a downstream mediator of adiponectin action [6].

Adiponectin has intriguing structural properties that may influence the in vivo activity of the protein. The native protein circulates in serum as lower molecular weight (LMW) hexamers and as larger multimeric structures of high molecular weight (HMW) [7]. HMW adiponectin consists of 1218 subunits and has been found to decline in concentration in mouse serum under administration of insulin and glucose. In fact, the ratio between HMW and LMW adiponectin, measured as the percentage of higher molecular weight adiponectin $\left(S_{\mathrm{A}}\right)$ index was found to be a better indicator of improved insulin sensitivity under thiazolidinedione treatment in both mice and humans [8].

Commercial assays readily measure the total plasma concentration of adiponectin. Here, we used such a commercial ELISA to measure total adiponectin. We also subjected the plasma to size fractionation and examined the relationship between total serum adiponectin and HMW as a percentage of total adiponectin (the $S_{\mathrm{A}}$ index) in a randomly selected cohort of patients with a range of glucose tolerance from normal to diabetic. This study was specifically designed to test whether subfractionation into adiponectin complexes offers superior correlations to 2-h glucose values following an oral glucose tolerance test compared with measurements of total adiponectin levels.

\section{Subjects, materials and methods}

Subjects A total of 34 Indo-Asian male subjects were randomly selected and included in the study (BMI 26.5 \pm $3.1 \mathrm{~kg} / \mathrm{m}^{2}$; age $52.15 \pm 10.14$ years). Each subject was fasted for $10 \mathrm{~h}$ before fasting blood was taken and a $75-\mathrm{g}$ oral glucose tolerance test was administered. From this, 2-h glucose values were determined in a laboratory using the glucose oxidase method. None of the subjects had been previously diagnosed with type 2 diabetes mellitus and were therefore not receiving any medication. Consent was obtained for all samples individually, in accordance with the requirements of the East Birmingham ethics committee.

Separation of adiponectin complexes Quantification of HMW and LMW adiponectin forms was performed as previously described [7]. Briefly, plasma was diluted $1: 5$ in $0.125 \mathrm{~mol} / 1 \mathrm{NaCl}, 0.01 \mathrm{~mol} / \mathrm{l}$ HEPES $\mathrm{pH} 8.0$ and layered onto $5-20 \%$ sucrose step gradients. These were spun at $55,000 \mathrm{rpm}$ for $4 \mathrm{~h}$ at $4^{\circ} \mathrm{C}$ and fractions $(150 \mu \mathrm{l})$ were retrieved sequentially from the top of the gradient and analysed by western blotting using an antibody specifically raised against the human aminoterminal hypervariable region of adiponectin [7]. Blots were treated with a ${ }^{125} \mathrm{I}-$ derivatized secondary goat anti-rabbit antibody (Amersham Biosciences, Chalfont St Giles, UK) and analysed with a Phosphoimager (Amersham Biosciences). Fractions 4-6 (LMW adiponectin) and 9-11 (HMW adiponectin) from velocity sedimentation were quantified with ImageQuant software (Amersham Biosciences UK, Bucks, England). $S_{\mathrm{A}}$ was calculated from raw densitometry values using (HMW/ $[\mathrm{HMW}+\mathrm{LMW}]) \times 100$.

Assessment of total adiponectin, insulin, IL-6 and leptin Total adiponectin and fasting insulin levels were determined using an ELISA-based colorimetric kit (Linco Research, St Charles, MO, USA) and leptin (ELISA; Linco Research). IL-6 levels were measured in one aliquot of serum using a singleplex cytokine panel (Bio-Rad, Hercules, CA, USA) and reported levels were determined using a Bioplex multiplex reader (Bio-Rad).

Statistical analysis of results All variables were initially analysed using bivariate Pearson correlation to address any relationship between the clinical parameters. Linear regression was used to assess the relationship between measurements of adiponectin and 2-h glucose levels. Residual plots were applied and all statistical outliers removed from the study. All $p$ values $<0.05$ were considered statistically significant. Analyses were carried out using the SPSS software package (SPSS, Chicago, IL, USA).

\section{Results}

Adiponectin and other parameters of glucose metabolism Fasting serum levels of adipose-derived proteins (leptin, adiponectin and IL-6) were analysed in 34 subjects. Fasting serum insulin and 2-h glucose were also recorded, along with BMI and the age of the individuals (Table 1). No correlation was observed between these markers and BMI in these individuals, whilst leptin $(r=0.340, p=0.049)$ and IL-6 $(r=0.353, p=0.041)$ were found to correlate positively with 2 -h glucose levels. In contrast, adiponectin displayed an inverse correlation $(r=0.378, p=0.028)$. Fasting insulin levels correlated with both adiponectin ( $r=$ $-0.356, p=0.039)$ and IL-6 $(r=0.366, p=0.033)$ but not leptin. Adiponectin was also associated with age $(r=0.566$, $p=0.0001$ ). Neither IL-6 nor leptin correlated with levels of total adiponectin or $S_{\mathrm{A}}$.

The $S_{A}$ index In all subjects the $S_{\mathrm{A}}$ index was analysed using velocity sedimentation and western blotting. There was a significant inverse correlation between 2-h glucose levels and $S_{\mathrm{A}}(r=-0.589, p=0.0001)$. In addition, there was

Table 1 Clinical and biochemical characteristics of the subjects

\begin{tabular}{lrc}
\hline & \multicolumn{1}{l}{ Range } & Mean \pm SEM \\
\hline Age $($ years $)(n=34)$ & $31.0-71.0$ & $52.1 \pm 1.8$ \\
BMI $\left(\mathrm{kg} / \mathrm{m}^{2}\right)$ & $21.0-32.0$ & $26.6 \pm 0.5$ \\
2-h glucose $(\mathrm{mmol} / \mathrm{l})$ & $4.3-20.7$ & $8.5 \pm 0.7$ \\
Total adiponectin $(\mu \mathrm{g} / \mathrm{ml})$ & $0.7-11.5$ & $5.2 \pm 0.5$ \\
$S_{\mathrm{A}}(\%)$ & $4.7-34.6$ & $17.6 \pm 1.3$ \\
Leptin $(\mathrm{ng} / \mathrm{ml})$ & $3.4-28.8$ & $10.0 \pm 0.95$ \\
Fasting insulin $(\mathrm{mU} / \mathrm{l})$ & $3.4-70.7$ & $17.2 \pm 2.3$ \\
IL-6 $(\mathrm{pg} / \mathrm{ml})$ & $8.9-85.3$ & $24.9 \pm 3.1$ \\
\hline
\end{tabular}




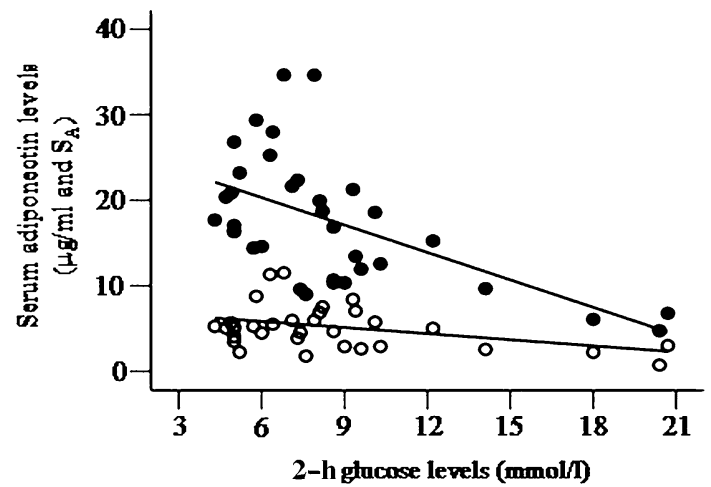

Fig. 1 Linear relationships between 2-h glucose level and $S_{\mathrm{A}}(y=$ $-1.069 x+26.71, r=-0.58, p=0.0003)$, shown as solid data points, and total adiponectin $(y=-0.238 x+7.250, r=-0.38, p=0.028)$ levels in serum, shown as ringed data points

a significant correlation between $S_{\mathrm{A}}$ and fasting insulin levels $(r=-0.354, p=0.040)$. Further analysis revealed that the levels of $S_{\mathrm{A}}$ also correlated with total adiponectin ( $r=$ $0.625, p=0.0001)$ and the age of the individual $(r=0.567$, $p=0.0001)$.

Total adiponectin versus HMW adiponectin Regression analysis (Fig. 1) displayed a strong linear relationship between $S_{\mathrm{A}}$ and 2-h glucose levels $(r=-0.58, p=0.0003)$, with an improved fit observed in the more severely glucose intolerant subjects. Total adiponectin levels did not appear to correlate as strongly with 2 -h glucose levels in this cohort $(r=-0.38, p=0.028)$. In addition, as both measurements had a similar correlation with age we did not adjust for this.

\section{Discussion}

This study was designed to specifically investigate the importance of $S_{\mathrm{A}}$ and the percentage of HMW adiponectin compared with the total measurement, made using a commercial ELISA. Our results show that, in these subjects, HMW adiponectin appeared to correlate strongly with 2-h glucose levels. This demonstrates the importance of HMW species of adiponectin as a possible regulator of in vivo insulin sensitivity and glucose uptake in humans. Our studies are in agreement with recent reports that HMW species are reduced in patients with type 2 diabetes mellitus [8]. Furthermore, a reduction in the serum level of HMW adiponectin was associated with the expected increase in fasting insulin level in the glucose-intolerant subjects. Studies have demonstrated that administration of insulin to mice causes a concurrent drop in circulating HMW adiponectin [7]. Further studies will, however, be needed to address the relationship between insulin and HMW adiponectin.

Other adipocyte-derived factors are thought to link obesity with glucose intolerance. Of these, IL-6 and leptin were considered in this study. IL-6 has been proposed as a potent regulator of adiponectin secretion [9] and sufficient quantities of leptin are thought to be required for normal adiponectin function. Although both were noted to associate positively with glucose intolerance in these individuals, neither correlated with $S_{\mathrm{A}}$ or total adiponectin.

Total adiponectin was negatively correlated with $2-\mathrm{h}$ glucose; however $S_{\mathrm{A}}$ showed a stronger relationship (Fig. 1). This suggests not only that $S_{\mathrm{A}}$ could act as a better marker of glucose intolerance but that HMW adiponectin is more likely to be functionally important for glucose tolerance and insulin sensitivity. These two measures correlate strongly with each other, which may lead us to postulate that any observed correlation with 2-h glucose in the total measurement may be due to the corresponding adjusted levels of HMW adiponectin. However, further investigation is required. Future studies will need to address whether low $S_{\mathrm{A}}$ values will predispose patients currently in the normal range towards future development of insulin resistance.

The role of total adiponectin in diabetic and glucoseintolerant subjects has been subject to intense scientific scrutiny. Many studies have contributed to the current view that this protein is a potent regulator of glucose and lipid metabolism. Our findings here support the emerging hypothesis that HMW adiponectin is the active form of the protein. This study was designed to accurately assess levels of HMW adiponectin. Although reproducible, the assay is laborious and not suited to the study of large cohorts. The challenge, therefore, is to produce an assay that allows a high throughput so that studies incorporating large numbers can be undertaken. Such an advance in technique would permit larger cohorts to be used, allowing statistical models to assess further the validity of $S_{\mathrm{A}}$ as a marker of glucose tolerance and insulin sensitivity in populations.

Acknowledgements f f. M. Fisher was supported by a European Foundation for the Study of Diabetes/MSD travel fellowship and a University of Birmingham studentship.

\section{References}

1. Comuzzie AG, Funahashi T, Sonnenberg G et al (2001) The genetic basis of plasma variation in adiponectin, a global endophenotype for obesity and the metabolic syndrome. J Clin Endocrinol Metab 86:4321-4325

2. Stefan N, Bunt JC, Salbe AD et al (2002) Plasma adiponectin concentrations in children: relationships with obesity and insulinemia. J Clin Endocrinol Metab 87:4652-4656

3. Hirose H, Kawai T, Yamamoto Y et al (2002) Effects of pioglitazone on metabolic parameters, body fat distribution, and serum adiponectin levels in Japanese male patients with type 2 diabetes. Metabolism 51:314-317

4. Hotta K, Funahashi T, Arita Y et al (2000) Plasma concentrations of a novel, adipose-specific protein, adiponectin, in type 2 diabetic patients. Arterioscler Thromb Vasc Biol 20:1595-1599

5. Kissebah AH, Sonnenberg GE, Myklebust J et al (2000) Quantitative trait loci on chromosomes 3 and 17 influence phenotypes of the metabolic syndrome. Proc Natl Acad Sci U S A 97:14478-14483

6. Yamauchi T, Kamon J, Minokoshi Y et al (2002) Adiponectin stimulates glucose utilization and fatty-acid oxidation by activating AMP-activated protein kinase. Nat Med 8:1288-1295 
7. Pajvani UB, Du X, Combs TP et al (2003) Structure-function studies of the adipocyte-secreted hormone Acrp30/adiponectin: implications for metabolic regulation and bioactivity. J Biol Chem 278:9073-9085

8. Pajvani UB, Hawkins M, Combs TP et al (2004) Complex distribution, not absolute amount of adiponectin, correlates with thiazolidinedione-mediated improvement in insulin sensitivity. J Biol Chem 279:12152-12162
9. Fasshauer M, Kralisch S, Klier M et al (2003) Adiponectin gene expression and secretion is inhibited by interleukin- 6 in 3T3-L1 adipocytes. Biochem Biophys Res Commun 301: $1045-1050$ 